\title{
QUISTE GIGANTE DE LA GLÁNDULA DE BARTOLINO: REPORTE DE CASO Y REVISIÓN DE LA LITERATURA
}

\section{A case report and literature review regarding giant Bartholin gland cyst}

Édgar E. Rivas-Perdomo, M.D.*

Recibido: diciembre 3/09 - Aceptado: noviembre 8/10

\section{RESUMEN}

Introducción: en el presente artículo se expone el caso de una paciente que tuvo un quiste gigante de la glándula de Bartolino, el cual alcanzó un diámetro de $10 \mathrm{~cm}$. La paciente presentó algunas dificultades diagnósticas y terapéuticas por lo que fue necesaria la escisión de la glándula y la posterior reconstrucción de la vulva. El diagnóstico definitivo fue establecido por anatomía patológica. El caso se presenta con el objetivo de realizar una revisión de la literatura respecto al tratamiento.

Materiales y métodos: se realizó una búsqueda de la literatura relacionada con el tema en las bases de datos electrónicas PubMed, SciELO, ScienceDirect y MD Consult, utilizando las palabras clave: "glándulas de Bartolino", "quistes" y "marsupialización”. Además, se revisaron referencias de libros de textos.

Conclusiones: la urgencia en el tratamiento depende de los síntomas de la paciente. Un quiste asintomático podría no requerir tratamiento. Sin embargo, la no mejoría dentro de las primeras 72 horas o la evolución hacia una forma de absceso requerirá un tratamiento quirúrgico de emergencia. Se ha descrito una amplia variedad de tratamientos que incluyen una serie de procedimientos

* Gineco-obstetra, Universidad de Cartagena. Clínica de Maternidad Rafael Calvo de Cartagena. Cartagena (Colombia). Correo electrónico: erivasperdomo@gmail.com quirúrgicos tales como: 1) incisión simple y drenaje, 2) fistulización o marsupialización, 3) colocación de un catéter de Word, 4) escleroterapia con alcohol, 5) aplicación de nitrato de plata y $\mathbf{6}$ ) ablación del quiste utilizando dióxido de carbono $\left(\mathrm{CO}_{2}\right)$ con láser.

Palabras clave: quiste gigante, glándula de Bartolino, quistes vaginales.

\section{SUMMARY}

Introduction: the case of a patient who had a giant Bartholin gland cyst is presented; it reached $10 \mathrm{~cm}$ diameter, presenting some diagnostic and therapeutic difficulties, requiring the excision of the gland and later reconstruction of the vulva. Definitive diagnosis was established by pathological anatomy. The case is presented as it led to a review of the pertinent literature regarding the relevant treatment.

Materials and methods: a search was made of the pertinent literature in PubMed, SciELO, ScienceDirect and MD Consult databases, using the key words "Bartholin glands", “cysts”, "marsupialization”. Pertinent reference books were also reviewed.

Conclusions: the urgency of treatment depends on a particular patient's treatment. An asymptomatic cyst may not require treatment; however, if there is no improvement within the first 72 hours or it evolves towards an abscess, then it will require emergency surgical treatment. 
A broad variety of treatments has been described which includes a series of surgical procedures, which include: simple incision and drainage, fistulization or marsupialization, placing a Word catheter, sclerotherapy with alcohol, applying silver nitrate and cyst ablation using carbon dioxide (CO2) laser.

Key words: giant cyst, Bartholin gland, vaginal cyst.

\section{INTRODUCCIÓN}

Los quistes de la glándula de Bartolino son problemas comunes en mujeres en edad reproductiva. La mayor ocurrencia está entre los 20 y 29 años de edad. ${ }^{1}$ Se estima que el $2 \%$ de las mujeres presenta algún episodio de quiste o absceso de la glándula de Bartolino durante el transcurso de la vida. ${ }^{2,3}$ Kondi-Pafiti y sus colegas reportan, en el 2008, una frecuencia del 28,5\% de quistes de la glándula de Bartolino en una serie de quistes vaginales benignos. ${ }^{4}$ Aunque los quistes suelen ser asintomáticos, pueden aumentar de tamaño o infectarse y causar un sufrimiento considerable con limitación de las actividades. ${ }^{5,6}$ La formación de quistes y abscesos resulta en una atrofia de la glándula y en muchos casos es innecesaria su preservación. ${ }^{7}$ En general, los quistes de las glándulas de Bartolino alcanzan diámetros de 1 a 4 cm; sin embargo, se informó sobre un caso en el que el quiste alcanzó $10 \mathrm{~cm}$ de diámetro. ${ }^{8}$ Los abscesos son tres veces más comunes que los quistes. Se ha señalado que las mujeres de raza blanca y de raza negra son más propensas a desarrollar quistes o abscesos de las glándulas de Bartolino en comparación con las mujeres hispanas y que la alta paridad se relaciona con un bajo riesgo para el mismo. ${ }^{9}$

La causa de la formación de los quistes está relacionada con la obstrucción distal del conducto de Bartolino, que resulta en una dilatación del mismo y en la formación de un quiste, el cual podría infectarse y generar un absceso de la glándula. Otra causa de los quistes son los traumas y cirugías vulvovaginales que, aunque en poca proporción, podrían generar estos quistes y abscesos. ${ }^{5,9} \mathrm{Se}$ estima que 1-2\% de las cirugías vulvovaginales podrían complicarse con quistes de las glándulas de Bartolino, ${ }^{10}$ dentro de las cuales se incluyen las episiorrafias mediolaterales. ${ }^{11}$ Durante una episiorrafia o una colporrafia posterior se podría lesionar o ligar el conducto. ${ }^{12}$

El diagnóstico diferencial debe considerar una serie de entidades tales como: los quistes de inclusión, quistes mucosos del vestíbulo, quistes de Nuck y quistes de las glándulas de Skene. En el caso de los abscesos de la glándula de Bartolino, un tercio de los pacientes puede presentar fiebre. ${ }^{9}$ Por otra parte, también se deben contemplar las lesiones sólidas como fibromas, lipomas, leiomiomas, neurofibroma, hernia inguinal que se extiende debajo del labio mayor e inclusive la posibilidad de cáncer., ${ }^{9,13}$ Se estima que en las mujeres mayores de 40 años se presenta un riesgo incrementado de malignidad de $2-7 \%$ de todas las lesiones malignas invasivas. ${ }^{5,9}$ Se ha informado sobre un caso de endometriosis localizado en la glándula de Bartolino. ${ }^{14}$

Existe una variedad de propuestas de tratamiento quirúrgico de la entidad con miras a disminuir las tasas de recurrencia. Además se observa una variedad de diagnósticos diferenciales con esta patología que incluye lesiones neoplásicas y que obliga a estudiar con detenimiento la presencia de masas en la zona genital. Al respecto, se presenta un caso con un crecimiento excesivo de un quiste de la glándula de Bartolino y una revisión de la literatura sobre aspectos relacionados con el tratamiento.

\section{PRESENTACIÓN DEL CASO}

Paciente femenino de 39 años de edad, soltera, que presenta cuadro de 10 años de evolución con masa en la zona del labio mayor derecho, no dolorosa, cuyo tamaño ha ido aumentado. Refiere la aparición de una masa similar y que fuera drenada con recurrencia desde el tiempo indicado. Antecedentes: asma, enfermedad ácido 
Figura 1. Masa genital.

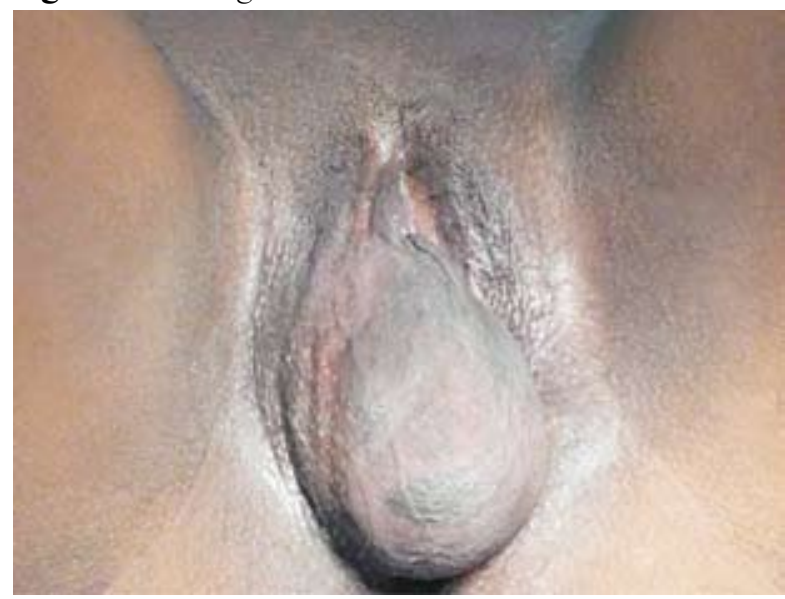

Figura 3. Quiste de la glándula de Bartolino.

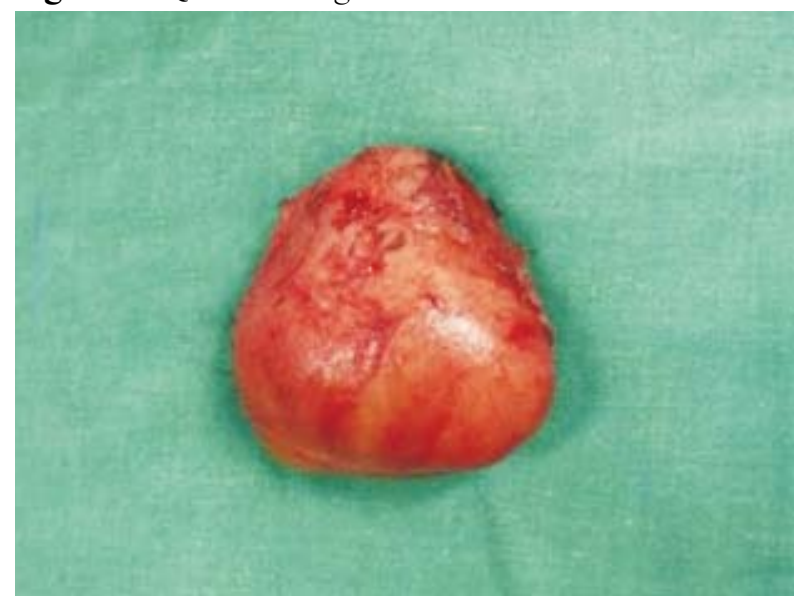

péptica, alergia a los AINEs; menarquia: 14 años; ciclos: 5x29; grávida: 4; paridad: 2 (cesáreas: 2); abortos: 2. Al momento del examen, el labio mayor derecho se encontraba con lesión blanda de $8 \mathrm{~cm}$ de diámetro aproximadamente, de consistencia blanda, móvil, no dolorosa, que cubre parcialmente el orificio vaginal (Figuras 1 y 2). Glándula de Bartolino izquierda no palpable. Vagina de longitud, amplitud y temperatura normales, con moderada cantidad de flujo blanco, grumoso y no maloliente. Cuello de aspecto sano. Útero de tamaño normal. Anexos no palpables. Resto del examen físico dentro de los límites normales. Paraclínicos: Hb: 10,5 g/dl; Hto: 31\%; leucocitos: 6900/ml; PMN: 38\%; linfocitos: 59\%; eosinófilos: 3\%; plaquetas: 350000/ml; TP: 13,8 s; TPT: $31 \mathrm{~s}$.
Figura 2. Masa genital. Vista de su extensión que ocluye el canal vaginal.

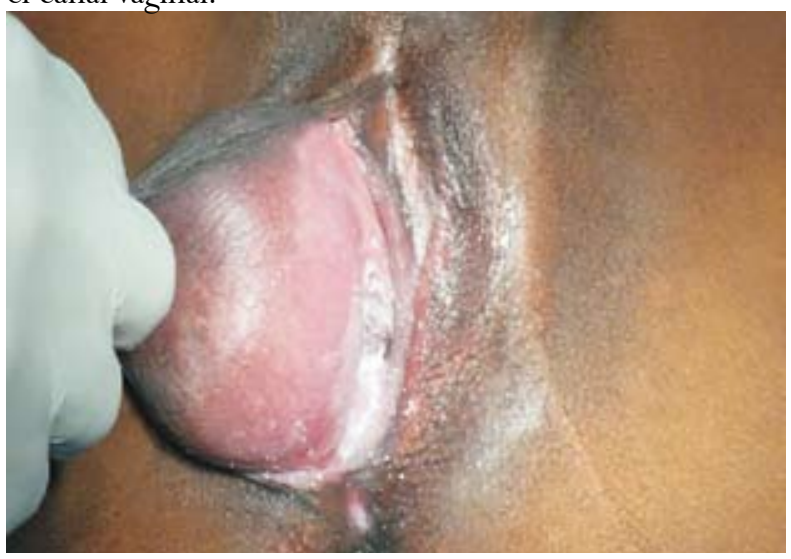

Figura 4. Reconstrucción vulvar posterior a la resección del quiste de la glándula de Bartolino.

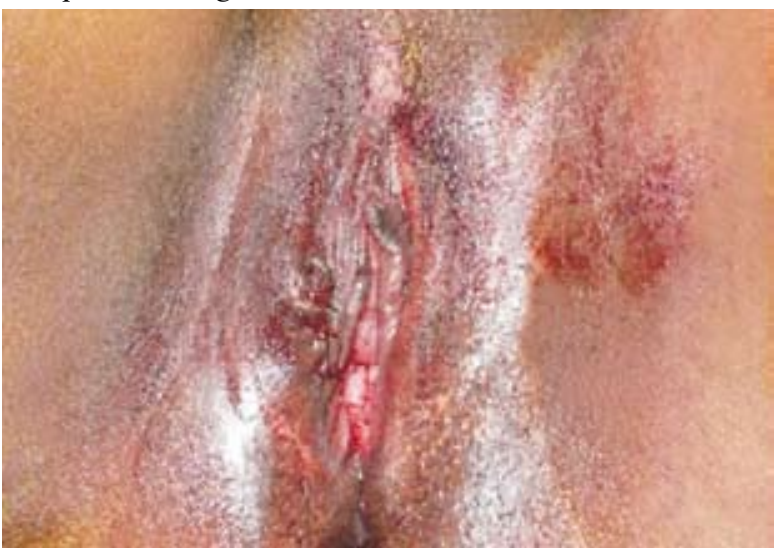

Se tienen como diagnósticos probables: quiste de la glándula de Bartolino derecha versus quiste de Nuck. Se realizó resección de la masa (figura 3) y posterior reconstrucción de la vulva (Figura 4). El informe de patología indica descripción macroscópica: "rotulado glándula de Bartolino se recibe una masa de 6,5 cm de diámetro mayor, la superficie irregular de color pardo grisáceo. Al corte hay salida de líquido de color pardo amarillento dejando una cavidad unilocular de paredes delgadas". Descripción microscópica: "los cortes revelan pared de una lesión quística tapizada por epitelio cilíndrico y epitelio escamoso maduro en otras áreas, que reposan en un estroma bien vascularizado. Diagnóstico final: quiste de la glándula de Bartolino. 


\section{MATERIALES Y MÉTODOS}

Se realizó una búsqueda de literatura relacionada con el tema en las bases de datos electrónicas MEDLINE vía PubMed, SciELO, ScienceDirect y MD Consult, utilizando las palabras clave: "glándulas de Bartolino", "quistes", "marsupialización". Se seleccionaron artículos de revisión, estudios primarios, series y reporte de casos. Además, se revisaron referencias de libros de textos.

\section{DISCUSIÓN}

El tratamiento quirúrgico del quiste de la glándula de Bartolino es similar al tratamiento del absceso de Bartolino y depende de los síntomas de la paciente. Un quiste asintomático podría no requerir tratamiento $;^{5}$ sin embargo, la no mejoría dentro de las primeras 72 horas o la evolución hacia una forma de absceso requerirá un tratamiento quirúrgico de emergencia. ${ }^{10}$ Se ha descrito una serie de procedimientos quirúrgicos tales como: 1) la fistulización o marsupialización, ${ }^{2} 2$ ) la colocación de un catéter de Word, ${ }^{5}$ 3) la escleroterapia con alcohol, ${ }^{11,15} 4$ ) incisión simple, drenaje ${ }^{15}$ 5) la aplicación de nitrato de plata, ${ }^{7,16}$ 6) la ablación del quiste utilizando dióxido de carbono $\left(\mathrm{CO}_{2}\right)$ con láser ${ }^{17}$ y 7$)$ la escisión de la glándula. ${ }^{5,10} \mathrm{~A}$ continuación se describen cada una de estas técnicas en detalle:

1) La marsupialización es presentada como el método adecuado para tratar los quistes y abscesos de las glándulas de Bartolino, por ser un procedimiento sencillo, rápido, poco doloroso y muy bien tolerado por la paciente. ${ }^{2}$ No obstante, la recurrencia es muy probable y sería necesaria la escisión quirúrgica. ${ }^{5}$ Se estima una recurrencia del 5 al 15\% de los quistes de Bartolino después de la marsupialización. Las complicaciones incluyen hematoma e infección. ${ }^{5}$ Es importante la cobertura antibiótica perioperatoria para gérmenes del tracto digestivo. Después de la cirugía se debe prescribir un antibiótico oral con un amplio espectro durante una semana. La paciente deberá ser revisada en consulta a los ocho días y posteriormente al mes. ${ }^{10}$
2) El drenaje definitivo implica la colocación del catéter de Word o la marsupialización. El catéter de Word, que se utiliza para el tratamiento de los quistes abscedados de las glándulas de Bartolino, es un dispositivo catéter de goma de 1 pulgada de largo y de diámetro de una sonda de Foley no 10F. La punta pequeña contiene un globo inflable que puede contener cerca de $3 \mathrm{ml}$ de solución salina que se inserta en la cavidad del quiste o absceso; el extremo libre podría dejarse dentro de la vagina. El objetivo es crear una fístula que se epiteliza en un lapso de 4 a 6 semanas, aunque ello generalmente ocurre en 3 a 4 semanas. $^{5}$

3) El principio de la escleroterapia por obliteración de la cavidad serosa de agentes como el alcohol, la tetraciclina, la bleomicina y el talco ha sido usado para estos propósitos en otras partes del cuerpo. ${ }^{18,19}$ En 2004, Kafali presenta un experimento clínico que evalúa la utilización del alcohol como agente esclerosante en quiste de la glándula de Bartolino, en comparación con la solución de nitrato. Después de la evacuación se regaron las cavidades con alcohol al 70\%, durante cinco minutos. La escleroterapia con alcohol se presenta como un procedimiento más rápido, con una cicatrización más rápida de la glándula, en comparación con el nitrato de plata. La recidiva se observó en el 8\% y el 10\% de los pacientes en los 2 estudios, a los 7 meses. ${ }^{11} \mathrm{La}$ actividad esclerosante se debe al $\mathrm{pH}$ ácido que causa una fuerte irritación de los tejidos. Esto, a su vez, ocasiona la destrucción de las células secretantes dentro del quiste y la adherencia de sus paredes. Ello se sucedería en un lapso de 1-3 minutos de contacto con el alcohol. ${ }^{11}$

4) Incisión simple, drenaje. Aunque produce una rápida mejoría, no es recomendable por la alta frecuencia de reincidencia. ${ }^{2}$

5) El nitrato de plata $\left(\mathrm{AgNO}_{3}\right)$ es un agente esclerosante y germicida que había sido utilizado para el tratamiento de condiciones quísticas en diferentes partes del cuerpo y se introdujo 
también para el tratamiento de los quistes de las glándulas de Bartolino. Destruye la pared del quiste o del absceso y en un tiempo suficiente es seguido de la formación de una fístula. El tratamiento con nitrato de plata tiene diversas ventajas: una sola aplicación es suficiente, con una duración de 5-10 minutos. La paciente se puede manejar ambulatoriamente con bajas tasas de fracasos. El dolor posoperatorio y la lesión a tejidos adyacentes es mínimo y no se altera la función sexual., ${ }^{7,16}$

6) El láser con dióxido de carbono $\left(\mathrm{CO}_{2}\right)$ también es considerado un método efectivo para el tratamiento de los quistes, con mínimo daño a los tejidos adyacentes, rápida curación y bajas tasas de recurrencia. ${ }^{2,20}$ Sin embargo, Hill y sus colegas consideran que el láser, por lo general, no ofrece ninguna ventaja sobre los procedimientos menos costosos y técnicamente menos difíciles descritos anteriormente. ${ }^{6}$ Con el fin de disminuir las probabilidades de recidivas anotadas, se ha considerado la aplicación de un agente esclerosante en la cavidad quística que induzca la adherencia de las paredes del mismo. De esta manera no sólo se elimina el contenido quístico, sino que también se destruye el revestimiento de la pared del quiste, se destruye la cavidad y se previene la reacumulación líquida. ${ }^{21}$

7) En algunos casos es necesaria la escisión de la glándula y podría ser considerada en pacientes que no responden a intentos conservadores de drenajes con fístulas. ${ }^{5,16} \mathrm{Si}$ el diagnóstico es dudoso, como en el caso de la paciente antes expuesto, se debe realizar la escisión. ${ }^{6}$ La escisión de la glándula de Bartolino es un procedimiento radical, que no debe realizarse durante el período agudo debido a los riesgos de hemorragia. ${ }^{10}$ Algunos investigadores recomiendan la escisión de la glándula de Bartolino cuando el quiste se presenta en pacientes mayores de 40 años. Aun cuando el adenocarcinoma de la glándula de Bartolino es raro, se debe considerar la referencia a oncología ginecológica en estos pacientes mayores. ${ }^{5}$
En general, existen múltiples tratamientos para los quistes de Bartolino. Sin embargo, según Wechter, al revisar la literatura se falla en no identificar el mejor enfoque terapéutico. Este autor concluye que se requieren ensayos clínicos controlados con un número suficiente de sujetos para poder disponer de una mejor evidencia. ${ }^{15}$

\section{CONCLUSIONES}

La urgencia en el tratamiento depende de los síntomas de la paciente. Un quiste asintomático podría no requerir tratamiento, pero la no mejoría dentro de las primeras 72 horas o la evolución hacia una forma de absceso requerirá un tratamiento quirúrgico de emergencia. Aunque se ha descrito una amplia variedad de tratamientos, no hay evidencia sólida que soporte el empleo de uno en especial.

\section{REFERENCIAS}

1. Cheetham DR. Bartholin's cyst: marsupialization or aspiration? Am J Obstet Gynecol 1985;152:569-70.

2. Pundir J, Auld BJ. A review of the management of disease of the Bartholin's gland. J Obstet Gynaecol 2008;28:161-5.

3. Eglin G. Comment je fais ... une bartholinectomie. Gynécologie [How I do ... Bartholin gland cystectomy] Obstétrique \& Fertilité 2007;35:1268-9.

4. Kondi-Pafiti A, Grapsa D, Papakonstantinou K, KairiVassilatou E, Xasiakos D. Vaginal cysts: a common pathologic entity revisited. Clin Exp Obstet Gynecol 2008;35:41-4.

5. Omole F, Simmons BJ, Hacker Y. Management of Bartholin's duct cyst and gland abscess. Am Fam Physician 2003;68:135-40.

6. Hill DA, Lense JJ. Office management of Bartholin gland cysts and abscesses. Am Fam Physician 1998;57:1611-6.

7. Mungan T, Ugur M, Yalqin H, Alan S, Sayilgan A. Treatment of Bartholin's cyst and abscess: excision versus silver nitrate insertion. Eur J Obstet Gynecol Reprod Biol 1995;63:61-3.

8. Adauy A, Salinas H, Naranjo B, Retamales B. Quistes vaginales. Rev chil obstet ginecol 2006;71:252-8.

9. Patil S, Sultan AH, Thakar R. Bartholin's cysts and abscesses. J Obstet Gynaecol 2007;27:241-5. 
10. Chêne G, Tardieu AS, Nohuz E, Rabischong B, Favard A, Mage G. Complications postopératoires des bartholinites aiguës. À propos de deux cas. [Postoperative complications of Bartholin's duct abscess. About two cases] Gynecol Obstet Fertil 2006;34:615-8.

11. Kafali H, Yurtseven S, Ozardali I. Aspiration and alcohol sclerotherapy: a novel method for management of Bartholin's cyst or abscess. Eur J Obstet Gynecol Reprod Biol 2004;112: 98-101.

12. Horowitz Ir, Buscema J, Majmudar B. Surgical conditions of the vulva. En: Rock JA, Jones HW III, editores. Te Linde's Operative Gynecology. 10 $10^{\mathrm{a}} \mathrm{ed}$. Lippincott William \& Wilkins; 2008. pp. 496-98.

13. Kusir VA, Mosquera C. Novel technique for management of Bartholin gland cysts and abscesses. J Emerg Med 2009;36:388-90.

14. Gocmen A, Inaloz HS, Sari I, Inaloz SS. Endometriosis in the Bartholin gland. European J Obstet Gynecol Reprod Biol 2004;114:110-1.

15. Wechter ME, Wu JM, Marzano D, Haefner H. Management of Bartholin duct cysts and abscesses: a systematic review. Obstet Gynecol Surv 2009; 64:395-404.
16. Turan C, Vicdan K, Gökmen O. The treatment of Bartholin's cyst and abscess with silver nitrate. Int J Gynecol Obstet 1995;48:317-8.

17. Marzano D, Haefner H. The Bartholin glan cyst: past, present, and future. J Low Genit Tract Dis 2004;8:195-204.

18. Spence J, Krings T, Terbrugge KG, Agid R. Percutaneous treatment of facial venous malformations: A matched comparison of alcohol and bleomycin sclerotherapy. Head Neck 2010 Apr 29. [Epub ahead of print].

19. Gatta G, Parlato V, Di Grezia G, Porto A, Cappabianca S, Grassi R, et al. Ultrasound-guided aspiration and ethanol sclerotherapy for treating endometrial cysts. Radiol Med 2010 Sep 17. [Epub ahead of print].

20. Penna C, Fambrini M, Fallani MG. $\mathrm{CO}_{2}$ laser treatment for Bartholin's gland cyst. Int J Gynecol Obstet 2002;76:79-80.

21. de Góis Speck NM, Belfort PN, Mendes P, Kang HJ, Ribalta JC. Carbon dioxide laser treatment of Bartholin's gland cyst. Clin Exp Obstet Gynecol 2007;34:50-1. 